\title{
Rosuvastatin, sildenafil and their combination in monocrotaline- -induced pulmonary hypertension in rat
}

\author{
MAGDALENA JASIŃSKA-STROSCHEIN* \\ JACEK OWCZAREK \\ ANNA WESOŁOWSKA \\ DARIA ORSZULAK-MICHALAK \\ Department of Biopharmacy \\ Medical University of Łódź \\ Łódź, Poland
}

\begin{abstract}
There is considerable interest in the pleiotropic effects of statins and their potential role in the treatment of pulmonary hypertension. Previous experimental findings indicate that a combination of lipophilic statins with phosphodiesterase type- 5 inhibitor, sildenafil, can offer preventive effects on rat monocrotaline-induced pulmonary hypertension. The present study is aimed to assess whether therapeutic regimen provides any benefits. Seven days after pulmonary hypertension induction, hydrophilic rosuvastatin and sildenafil were given for 14 days to male Wistar outbred rats. Right ventricular pressure, right ventricle mass and three biomarkers were evaluated after 21 days: brain natriuretic peptide, high-density lipoprotein cholesterol and vascular endothelial growth factor. The present study demonstrates that administration of hydrophilic statin with sildenafil results in reduction of pulmonary vascular remodeling and right ventricular pressure. The results of biochemical measurements may suggest that statins play a positive role in right ventricle function or the process of angiogenesis in pulmonary hypertension development.
\end{abstract}

Keywords: rosuvastatin, sildenafil, combination therapy, pulmonary hypertension, hemodynamic parameters

HMG-CoA reductase inhibitors (statins) are well-known for their pleiotropic effects which go beyond serum cholesterol lowering activity $(1,2)$. It has been demonstrated that statins might offer preventive or therapeutic benefits in animal models of pulmonary hypertension $(\mathrm{PH})(3,4)$. Such effects, primarily concerned with improving or restoring endothelial function, inhibiting the proliferation and migration of vascular smooth muscle cells or reducing myocardial mass and fibrosis (5), have been demonstrated mainly for simvastatin, one of the most "popular « statins, with pleiotropic activity being confirmed clinically in a number of multicenter trials (6). The influence of other statins, including less lipophilic ones, has only been assessed in very few experimental studies (7-10). Satoh et al. (7) did not observe 28-d pravastatin administration to have any significant impact on the reduction of elevated right ventricular pressure (RVP) and cardiac hypertrophy in a mono-

\footnotetext{
* Correspondence; e-mail: magdalena.jasinska-stroschein@umed.lodz.pl
} 
crotaline (MCT) rat model. In opposite, Sun et al. (8) showed that another hydrophilic statin - rosuvastatin when given especially for early protection against PH development had positive impact on myocardial hypertrophy and reduction of pulmonary pressure. Also, the benefits of adding statins to a conventional regimen have been investigated in several studies. These have usually included lipophilic statins, such as simvastatin or atorvastatin, and the drugs were administered just after induction of pulmonary hypertension as a preventive regimen. The results of such previous studies vary. Kuang et al. (11) showed sildenafil to have further benefits in a preventive model if co-administered with simvastatin. Zhao et al. (12) confirmed the beneficial impact of therapeutic regimen of statin on further reduction in right ventricle hypertrophy $(\mathrm{RVH})$ and vascular remodeling when added to sildenafil therapy. However, the obtained results were not accompanied by further reduction of pulmonary arterial pressure (PAP) as compared to sildenafil alone (12). Other studies have not demonstrated any such hemodynamic benefits when statin was added to conventional - sildenafil (13) or beraprost (7) therapy. Based on these reports, the present study was undertaken to assess whether hydrophilic HMG-CoA reductase inhibitor could provide greater effects if added to a conventional PH regimen. A MCT rat model was used to evaluate the effects of the combined usage of rosuvastain with sildenafil, an agent known to be effective in reducing pulmonary hypertension. The study was addressed to therapeutic not preventive regimen, i.e., drugs were administered after pulmonary hypertension development. The effects of such treatment were assessed in relation to the normalization of RVP and to the right ventricle mass, especially. The study includes some circulating markers regarded as being associated with $\mathrm{PH}$, as several are known to have prognostic potential and show promise for monitoring the response to $\mathrm{PH}$ therapy. These are high-density lipoprotein cholesterol (HDL-C), brain natriuretic peptide (BNP) and vascular endothelial growth factor A (VEGF-A).

\section{EXPERIMENTAL}

\section{Animals}

The experiments were performed on thirty-eight outbred Wistar male rats, body mass 230-400 g, fed on granulated Murigran (Agropol S. J., Poland), with free access to water. The animals were housed in standard cages, five animals per cage. The experimental procedures were carried out in accordance with the international guidelines for care and use of laboratory animals. All efforts were made to minimize animal suffering and reduce the number of animals used in the experiments. All the procedures in these experiments were approved by the Ethics Committee of the Medical University of Lodz, Poland.

All drugs, in doses detailed in previous experiments on rats $(14,15)$, were administered by oral gavage, intragastrically (i.g.) suspended in $1.0 \%$ methylcellulose (MC).

Monocrotaline treatment. - Monocrotaline (MCT, Fluorochem Ltd, UK) was dissolved in $1 \mathrm{~mol} \mathrm{~L}^{-1} \mathrm{HCl}$, and $\mathrm{pH}$ was adjusted to 7.4 with $1 \mathrm{~mol} \mathrm{~L}^{-1} \mathrm{NaOH}$ (15). Monocrotaline was administered as a single, subcutaneous (s.c.) injection $\left(60 \mathrm{mg} \mathrm{kg}^{-1}\right)$ in a volume of $3 \mathrm{~mL} \mathrm{~kg}^{-1}$. Control, age-matched rats received an equal volume of vehicle. 
Experimental design. - The animals were divided into five groups of rats, randomly allocated to each group: 1) healthy rats receiving $1.0 \%$ methylcellulose, $1 \mathrm{~mL} \mathrm{~kg}^{-1}$, i.g. daily for 14 days $(n=9)$ - control group; 2 ) monocrotaline-induced rats receiving $1.0 \%$ methylcellulose, $1 \mathrm{~mL} \mathrm{~kg}^{-1}$, i.g. daily for 14 days after single, subcutaneous monocrotaline injection - MCT ( $n=8) ; 3$ ) monocrotaline-induced rats receiving rosuvastatin $10 \mathrm{mg} \mathrm{kg}^{-1}$, i.g. daily for 14 days $(n=8)$ - MCT+ROS; 4$)$ monocrotaline-induced rats receiving sildenafil $25 \mathrm{mg} \mathrm{kg}^{-1}$ i.g. daily for 14 days $(n=6)-\mathrm{MCT}+\mathrm{SIL} ; 5)$ monocrotaline-induced rats receiving rosuvastatin $10 \mathrm{mg} / \mathrm{kg}+$ sildenafil $25 \mathrm{mg} \mathrm{kg}^{-1}$, i.g. daily for 14 days $(n=7)-\mathrm{MCT}+\mathrm{ROS}+\mathrm{SIL}$. Rats were weighed once a week. The first dose of drug in the following group of animals: MCT+ROS, MCT+SIL, MCT+ROS+SIL was given 7 days after MCT injection.

Hemodynamic studies. - The surgery was performed 24 hours after the administration of the last drug dose. The rats were placed in dorsal position on the operation table. The animals were anaesthetized with penthobarbital sodium $\left(30 \mathrm{mg} \mathrm{kg}^{-1}\right)$, administered intraperitoneally. Anesthesia was maintained by additional bolus doses of penthobarbital sodium as needed. Lidocaine was used for local infiltration of the surgical sites. The registration of hemodynamic parameters in rats was performed by using a Hugo Sachs Electronik Haemodyn (Harvard Apparatus GmbH, Germany), as described previously (16).

Measurement of organ mass. - At the end of the study, after animal euthanasia, the heart was excised. Next, the right ventricle, right atrium and left ventricle plus septum were separated and weighed. The ratio of the right ventricle weight to left ventricle plus septum weight $(\mathrm{RV} / \mathrm{LV}+\mathrm{S})$ as a index of right ventricular hypertrophy was calculated.

Measurement of HDL-C, VEGF-A and BNP levels. - The levels of BNP and VEGF-A were measured in plasma samples with commercially available ELISA kits for rats (Life Science Inc., USA) and HDL-C was measured in plasma samples with reagents provided by Biolabo Reagents, France.

\section{Statistical analysis}

The statistical analysis was carried out using the Statgraphics Plus 5.0. The statistical evaluation was performed using the analysis of variance (ANOVA) and post-hoc comparisons were performed using the Student-Newman-Keuls test. Normal distribution of a parameter was checked by means of the Kolmogorov-Smirnov test with the Lillieforce correction. The homogeneity of variance was tested by Levene's test. All parameters were considered statistically significantly different if $p<0.05$.

\section{RESULTS AND DISCUSSION}

\section{Survival and hemodynamic parameters and cardiac hypertrophy}

At the end of the study, no rats died in the control as well as monocrotaline and other experimental groups.

A single subcutaneous injection of MCT resulted in significant increase in right ventricular pressure (RVP) compared to saline injected control animals $(p<0.05)$. The 14-day 

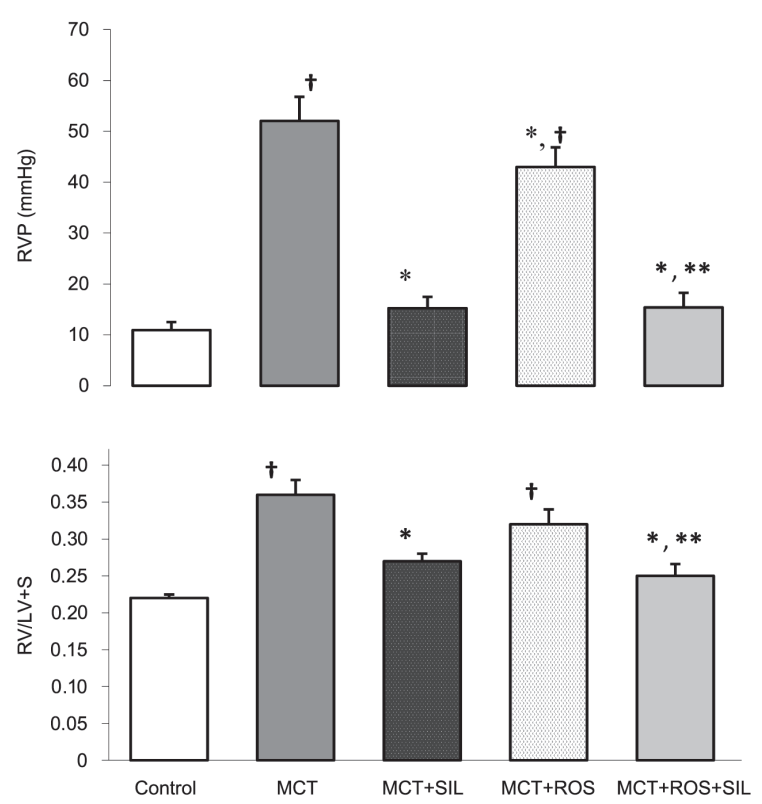

Fig. 1. Right ventricular pressure (RVP) and right ventricular hypertrophy expressed as the ratio of the right ventricular mass to left ventricle plus septum mass $(\mathrm{RV} / \mathrm{LV}+\mathrm{S})$ in the following groups of rats: control; monocrotaline - MCT; sildenafil - MCT+SIL; rosuvastatin - MCT+ROS; rosuvastatin + sildenafil - MCT+ROS+SIL. Results expressed as mean $\pm \mathrm{SE}, n=38$. Significant difference: ${ }^{*} p<0.05$ as compared to monocrotaline (MCT) group, ${ }^{* *} p<0.05$ as compared to rosuvastatin group, $\boldsymbol{\dagger} p<0.05$ as compared to control group.

administration of sildenafil at the dose of $25 \mathrm{mg} \mathrm{kg}^{-1}$ or rosuvastatin at the dose of $10 \mathrm{mg}$ $\mathrm{kg}^{-1}$ reduced RVP significantly compared to MCT treated animals $(p<0.05)$. Fourteen-day administration combined administration of rosuvastatin and sildenafil caused significant decrease in RVP parameter compared to monocrotaline $(p<0.05)$ and to rosuvastatin alone $(p<0.05)$ (Fig. 1).

No significant difference regarding values of heart rate, systolic, mean and diastolic blood pressure among examined groups of animals was noted.

The ratio of the right ventricle mass to left ventricle plus septum mass (RV/LV+S) was significantly higher in MCT treated animals than in control group $(p<0.05)$. The administration of sildenafil alone caused significant decrease of this ratio $(p<0.05)$. Also, combined administration of sildenafil and rosuvastatin resulted in significant decrease in RV/LV+S ratio compared to MCT rats $(p<0.05)$ and to rosuvastatin alone $(p<0.05)$ (Fig. 1$)$.

\section{Biochemical parameters}

Administration of rosuvastatin and sildenafil alone or in combination did not cause any significant changes in plasma HDL-C level compared to MCT treated rats or saline group (Fig. 2). 
In monocrotaline treated rats plasma VEGF-A level was significantly lower compared to saline control group $(p<0.05)$. Administration of rosuvastatin or sildenafil alone caused a significant increase in plasma VEGF-A compared to MCT treated rats $(p<0.05)$. Combined, sildenafil plus rosuvastatin, administration caused increase in VEGF-A level compared to rats exposed to MCT ( $p=0.0502)$ (Fig. 2).

Monocrotaline induction caused significant increase in plasma BNP level compared to saline control group $(p<0.05)$. Administration of rosuvastatin or sildenafil alone caused
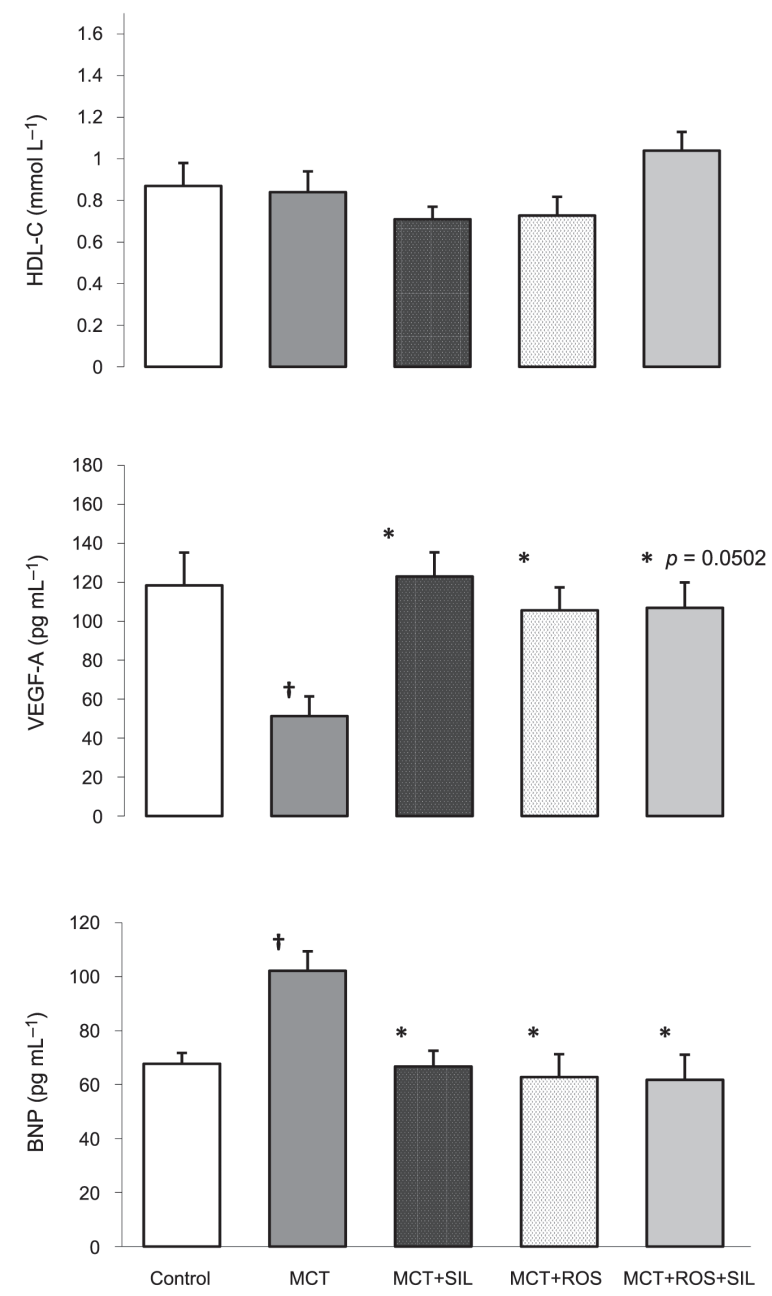

Fig. 2. Plasma HDL-C, VEGF-A and BNP levels in groups of rats: control, monocrotaline - MCT, sildenafil - MCT+SIL, rosuvastatin - MCT+ROS, rosuvastatin + sildenafil - MCT+ROS+SIL. Results expressed as mean $\pm \mathrm{SE}, n=38$. Significant difference: ${ }^{*} p<0.05$ as compared to monocrotaline (MCT) group, $\boldsymbol{\dagger} p<0.05$ as compared to control group. 
a significant decrease in plasma BNP level compared to MCT treated rats $(p<0.05)$; the same is true for combined sildenafil and rosuvastatin administration (Fig. 2).

The current approach to the treatment of pulmonary hypertension is to combine drugs from different classes to achieve greater therapeutic effects (17). This study is the first to present the "added « effects of a therapeutic regimen of hydrophilic rosuvastatin co-administered with sildenafil, agent with confirmed efficacy in $\mathrm{PH}$, as opposed to a preventive one. In the present study each drug significantly reversed a MCT-induced increase in RVP, however, their combined usage brought beneficial therapeutic but not additive results. In the study hydrophilic rosuvastatin was found to have moderate but significant effects, as similarly noted by Pei et al. (9). These authors observed rosuvastatin-induced modulation of right ventricular function, normalization of right ventricular hypertrophy as well as remodeling the small pulmonary artery; these effects were mediated through regulating the protein kinase Akt and endothelial nitric oxide synthase (Akt/eNOS) signaling pathway. As mentioned above, previous experimental research showed sildenafil to have further benefits in a preventive model if co-administered with simvastatin (11). Simvastatin and sildenafil are both substrates for the CYP3A4 isoenzyme. The potential competitive inhibition of drug metabolism might explain the further enhancement of sildenafil activity in a study by Kuang et al. (11). The opportunity of greater exposure being offered by a combination of sildenafil and simvastatin was also discussed by authors of clinical trial (SIPTH) (18). However, although such a mechanistic explanation could be attributed to sildenafil-simvastatin, the same cannot be said for the sildenafil-rosuvastatin interaction. In contrast to the lipophilic simvastatin (19), the hydrophilic rosuvastatin is a poor substrate of CYP3A4 without inhibitory activity towards cytochrome P450 isoenzymes. Nevertheless, recent clinical trials demonstrate only transient efficacy when phosphodiesterase type 5 inhibitor and/or endothelin receptor antagonist were supplemented by statins in PH (18). Such disappointing results have raised i.e., the question of whether particular statins at particular doses or regimens will be effective rather than all statins $(18,20)$. The time when statin is added to $\mathrm{PH}$ conventional therapy might also influence its efficacy. Although the findings of the present study, and those of earlier studies, imply that co-administration of statin with conventional drugs could confer advantages in treating $\mathrm{PH}$, especially for its early protection, further studies are needed to confirm this.

The results of the present study also indicate that rosuvastatin therapy, administered as monotherapy or in combination with sildenafil, exerts a significant impact on the changes of several factors which act as prognostic biomarkers in PH. Although this is confirmed for BNP, it is only discussed for VEGF-A. The role of such factors in pulmonary hypertension, together with several mechanistic implications, has been previously discussed (16).

The present study is first to demonstrate that a therapeutic statin regimen exerts a beneficial impact on reduction of BNP levels being elevated in MCT-induced PH. The benefits of statin therapy on right ventricle function with BNP level reductions have been described in heart failure or myocardial infarction (21, 22). Our observations suggest that statins might also play a positive role by ameliorating increased wall stress in the right ventricle in pulmonary hypertension. Very little data is available regarding statin impact on angiogenesis with VEGF changes resulting in pulmonary hypertension development. Chen et al. (23) observed that VEGF mRNA expression levels were increased in the lungs of a dehydromonocrotaline group, and these increases were reduced towards normal levels in the statin-treated group. However, our findings show that plasma VEGF levels were 
significantly higher in treated and saline control groups as compared to the monocrotaline group, and these observations were similar to the results of other treatment regimens (bosentan, sildenafil or iloprost) as described by Yigitaslan et al. (24). The authors concluded that lower serum and pulmonary artery VEGF levels in MCT group compared to treated ones may suggest the protective function of VEGF in early PH phases. However, further studies to evaluate the precise mechanistic linkage are needed.

There are some limitations of the study. First, mean pulmonary arterial pressure or cardiac output were not assessed. As previous experiments on PH animal models show, drug-induced alterations in RVP tend to reflect changes of pulmonary artery hemodynamics $(11,25)$. Secondly, the effects on pulmonary vessels could demonstrate the antiremodeling properties of proposed regimens, in addition to the hemodynamics and right ventricle hypertrophy results.

\section{CONCLUSIONS}

In conclusion, the present preliminary study shows that therapeutic regimen of a hydrophilic statin, rosuvastatin, can reverse MCT-induced pulmonary hypertension in rats in a moderate, but significant, manner by reducing pulmonary vascular remodeling and right ventricular pressure.

The results of biochemical measurements may suggest that statin plays a positive role towards right ventricle function or angiogenesis process in $\mathrm{PH}$ development. However, no additive effect of combination therapy with sildenafil was demonstrated. Further studies are needed to assess statin benefits in pulmonary hypertension in relation to the impact of study regimen (i.e., therapeutic vs. preventive), the potential effects of other drug-statin interactions and their mechanistic background.

Acronyms. BNP - brain natriuretic peptide, eNOS - endothelial nitric oxide synthase, HDL-C high-density lipoprotein cholesterol, MC - methylcellulose, MCT - monocrotaline, $\mathrm{PH}$ - pulmonary hypertension, PAP - pulmonary arterial pressure, ROS - rosuvastatin, $\mathrm{RVH}$ - right ventricle hypertrophy, $\mathrm{RV} / \mathrm{LV}+\mathrm{S}$ - ratio of the right ventricle mass to left ventricle plus septum mass, RVP - right ventricular pressure, SIL - sildenafil, VEGF - vascular endothelial growth factor

Acknowledgements. - The study was supported by the Medical University of Łódź, grant no. 50203/3-011-02/502-34-028; 503/3-011-02/503-01, 503/3-011-02/503-06-300.

\section{REFERENCES}

1. J. K. Liao and U. Laufs, Pleiotropic effects of statins, Annu. Rev. Pharmacol. Toxicol. 45 (2005) 89-118; DOI: 10.1146/annurev.pharmtox.45.120403.095748.

2. S. Wolfrum, K. S. Jensen and J. K. Liao, Endothelium-dependent effects of statins, Arterioscler. Thromb. Vasc. Biol. 23 (2003) 729-736; DOI: 10.1161/01.ATV.0000063385.12476.A7.

3. L. Taraseviciene-Stewart, R. Scerbavicius, K. H. Choe, C. Cool, K. Wood, R. M. Tuder, N. Burns, M. Kasper and N. F. Voelkel, Simvastatin causes endothelial cell apoptosis and attenuates severe pulmonary hypertension, Am. J. Physiol. Lung Cell Mol. Physiol. 291 (2006) L668-L676; DOI: 10.1152/ ajplung.00491.2005. 
4. R. E. Girgis, D. Li, X. Zhan, J. G. Garcia, R. M. Tuder, P. M. Hassoun and R. A. Johns, Attenuation of chronic hypoxic pulmonary hypertension by simvastatin, Am. J. Physiol. Heart Circ. Physiol. 285 (2003) H938-945; DOI: 10.1152/ajpheart.01097.2002.

5. F. Simko, Statins: a perspective for left ventricular hypertrophy treatment, Eur. J. Clin. Invest. 37 (2007) 681-691; DOI: 10.1111/j.1365-2362.2007.01837.x.

6. P. J. Delahoy, D. J. Magliano, K. Webb, M. Grobler and D. Liew, The relationship between reduction in low-density lipoprotein cholesterol by statins and reduction in risk of cardiovascular outcomes: an updated meta-analysis, Clin. Ther. 31 (2009) 236-244; DOI: 10.1016/j.clinthera.2009.02.017.

7. M. Satoh and A. Satoh, 3-Hydroxy-3-methylglutaryl (HMG)-COA reductase inhibitors and phosphodiesterase type $\mathrm{V}$ inhibitors attenuate right ventricular pressure and remodeling in a rat model of pulmonary hypertension, J. Pharm. Pharm. Sci. 11 (2009) 118s-130s.

8. X. Sun and D. D. Ku, Rosuvastatin provides pleiotropic protection against pulmonary hypertension, right ventricular hypertrophy, and coronary endothelial dysfunction in rats, Am. J. Physiol. Heart Circ. Physiol. 294 (2008) H801-H809; DOI: 10.1152/ajpheart.01112.2007.

9. Y. Pei, P. Ma, X. Wang, W. Zhang, X. Zhang, P. Zheng, L. Yan, Q. Xu and G. Dai, Rosuvastatin attenuates monocrotaline-induced pulmonary hypertension via regulation of Akt/eNOS signaling and asymmetric dimethylarginine metabolism, Eur. J. Pharmacol. 666 (2011) 165-172; DOI: 10.1016/j. ejphar.2011.05.035.

10. V. G. DeMarco, J. Habibi, A. T. Whaley-Connell, R. I. Schneider, J. R. Sowers, B. T. Andresen, A. A. Gutweiler, L. Ma, M. S. Johnson, C. M. Ferrario and K. C. Dellsperger, Rosuvastatin ameliorates the development of pulmonary arterial hypertension in the transgenic (mRen2)27 rat, Am. J. Physiol. Heart Circ. Physiol. 297 (2009) H1128-1139; DOI: 10.1152/ajpheart.00048.2009.

11. T. Kuang, J. Wang, B. Pang, X. Huang, E. D. Burg, J. X. Yuan and C. Wang, Combination of sildenafil and simvastatin ameliorates monocrotaline-induced pulmonary hypertension in rats, Pulm. Pharmacol. Ther. 23 (2010) 456-464; DOI: 10.1016/j.pupt.2010.02.003.

12. L. Zhao, A. Sebkhi, O. Ali, B. Wojciak-Stothard, L. Mamanova, Q. Yang, J. Wharton and M. R. Wilkins, Simvastatin and sildenafil combine to attenuate pulmonary hypertension, Eur. Respir. J. 34 (2009) 948-957; DOI: 10.1183/09031936.00143508.

13. D. S. Lee, Y. K. Kim and Y. W. Jung, Simvastatin, sildenafil and their combination in monocrotaline induced pulmonary arterial hypertension, Korean Circ. J. 40 (2010) 659-664; DOI: 10.4070/ kcj.2010.40.12.659.

14. X. L. Li, R. J. Guan, Q. H. Xu and Z. Y. Wu, [Effects of rosuvastatin on monocrotaline-induced pulmonary artery hypertension in rats], Zhonghua Xin Xue Guan Bing Za Zhi. 39 (2011) 247-253.

15. R. T. Schermuly, K. P. Kreisselmeier, H. A. Ghofrani, A. Samidurai, S. Pullamsetti, N. Weissmann, C. Schudt, L. Ermert, W. Seeger and F. Grimminger, Antiremodeling effects of iloprost and the dual-selective phosphodiesterase 3/4 inhibitor tolafentrine in chronic experimental pulmonary hypertension, Circ. Res. 94 (2004) 1101-1108; DOI: 10.1161/01.RES.0000126050.41296.8E.

16. M. Jasińska-Stroschein, J. Owczarek, A. Łuczak, and D. Orszulak-Michalak, The beneficial impact of fasudil and sildenafil on monocrotaline-induced pulmonary hypertension in rats: A hemodynamic and biochemical study, Pharmacology 91 (2013) 178-184; DOI: 10.1159/000346921.

17. N. Galiè, M. M. Hoeper, M. Humbert, A. Torbicki, J. L. Vachiery, J. A. Barbera, M. Beghetti, P. Corris, S. Gaine, J. S. Gibbs, M. A. Gomez-Sanchez, G. Jondeau, W. Klepetko, C. Opitz, A. Peacock, L. Rubin, M. Zellweger and G. Simonneau, ESC Committee for Practice Guidelines (CPG): Guidelines for the diagnosis and treatment of pulmonary hypertension: the task force for the diagnosis and treatment of pulmonary hypertension of the European Society of Cardiology (ESC) and the European Respiratory Society (ERS), endorsed by the International Society of Heart and Lung Transplantation (ISHLT), Eur. Heart. J. 30 (2009) 2493-2537; DOI: 10.1093/eurheartj/ehp297. 
18. M. R. Wilkins, O. Ali, W. Bradlow, J. Wharton, A. Taegtmeyer, C. J. Rhodes, H. A. Ghofrani, L. Howard, P. Nihoyannopoulos, R. H. Mohiaddin and J. S. Gibbs, Simvastatin Pulmonary Hypertension Trial (SiPHT) Study Group, Simvastatin as a treatment for pulmonary hypertension trial, Am. J. Respir. Crit. Care Med. 181 (2010) 1106-1113; DOI: 10.1164/rccm.2009111-699OC.

19. C. Transon, T. Leemann and P. Dayer, In vitro comparative inhibition profiles of major human drug metabolising cytochrome P450 isozymes (CYP2C9, CYP2D6 and CYP3A4) by HMG-CoA reductase inhibitors, Eur. J. Clin Pharmacol. 50 (1996) 209-215.

20. W. J. Zeng, C. M. Xiong, L. Zhao, G. L. Shan, Z. H. Liu, F. Xue Q. Gu, X. H. Ni, Z. H. Zhao, X. S. Cheng, M. R. Wilkins and J. G. He, Atorvastatin in pulmonary arterial hypertension (APATH) study, Eur. Respir. J. 40 (2012) 67-74; DOI: 10.1183/09031936.00149011.

21. E. Abulhul, K. McDonald, R. Martos, D. Phelan, J. P. Spiers, M. Hennessy, J. Baugh, C. Watson, C. O'Loughlin and M. Ledwidge, Long-term statin therapy in patients with systolic heart failure and normal cholesterol: effects on elevated serum markers of collagen turnover, inflammation, and B-type natriuretic peptide, Clin. Ther. 34 (2012) 91-100; DOI: 10.1016/j.clinthera.2011.11.002.

22. Y. Teshima, K. Yufu, H. Akioka, T. Iwao, F. Anan, M. Nakagawa, H. Yonemochi, N. Takahashi, M. Hara and T. Saikawa, Early atorvastatin therapy improves cardiac function in patients with acute myocardial infarction, J. Cardiol. 53 (2009) 58-64; DOI: 10.1016/j.jjcc.2008.08.008.

23. D. Chen, D. Zhou, J. Qian, F. Chen, L. Guan, L. Dong and J. Ge, Atorvastatin prevents dehydromonocrotaline-induced pulmonary hypertension in beagles, Exp Lung Res. 38 (2012) 333-343; DOI: 10.3109/01902148.2012.702852.

24. S. Yigitaslan and B. Sirmagul, Relation of bosentan, iloprost, and sildenafil with growth factor levels in monocrotaline-induced pulmonary hypertension, Clin. Exp. Hypertens. 34 (2012) 222-229; DOI: 10.3109/10641963.2011.631652.

25. K. T. Mouchaers, I. Schalij, M. A. de Boer, P. E. Postmus, V. W. van Hinsbergh, G. P. van Nieuw Amerongen, A. Vonk Noordegraaf and W. J. van der Laarse, Fasudil reduces monocrotaline-induced pulmonary arterial hypertension: comparison with bosentan and sildenafil, Eur. Respir. J. 36 (2010) 800-807; DOI: 10.1183/09031936.00130209. 